\title{
La cápsula endoscópica como estudio diagnóstico en gastroenterología
}

\author{
Capsule endoscopy as a diagnostic method in gastroenterology
}

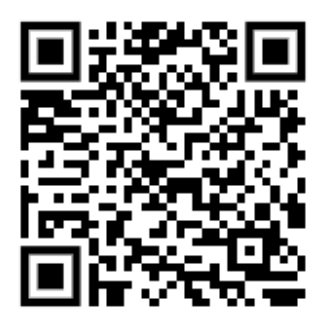

${ }^{1}$ Médico general, graduada de la Universidad de Costa Rica (UCR), médico investigadora independiente, San José, Costa Rica.

\author{
${ }^{1}$ Dra. María Fernanda Lynch Mejía \\ Investigadora independiente, San José, Costa Rica \\ mariafernandalynch@gmail.com \\ https://orcid.org/0000-0002-9049-5021
}

RECIBIDO

CORREGIDO

ACEPTADO

$28 / 01 / 2019$

$15 / 02 / 2019$

$15 / 03 / 2019$

\section{RESUMEN}

La cápsula endoscópica es un método diagnóstico de lesiones del intestino delgado, un órgano que clásicamente ha sido de difícil acceso por los estudios diagnósticos convencionales.

Su principal indicación es para el estudio de sangrado digestivo de origen oculto.

El siguiente artículo informativo expone las generalidades de los aspectos técnicos, indicaciones, contraindicaciones y preparación para el estudio por capsula endoscópica.

También se expone una revisión de las principales entidades diagnosticadas y los nuevos avances en endoscopía capsular para la detección de patologías en colon y esófago.

PALABRAS CLAVES: endoscopía capsular; intestino delgado; enfermedad de Crohn; hemorragia gastrointestinal

\section{ABSTRACT}

Capsule endoscopy is a diagnostic method to detect lesions of the small intestine, an area that has traditionally been difficult to access by conventional diagnostic studies.

The main indication for capsule endoscopy is obscure gastrointestinal hemorrhage. The following informative article summarizes the technical aspects, indications, contraindications and preparation for capsule endoscopy use.

There is also a review of the main diseases diagnosed and new advances in capsule endoscopy for the detection of pathologies in the colon and esophagus. 
KEYWORDS: capsule endoscopy; small intestine; Crohn's disease; gastrointestinal hemorrhage.

\section{INTRODUCCIÓN}

El intestino delgado es un área del tracto digestivo con difícil acceso por parte de los métodos de estudio gastroenterológicos clásicos (1, 2, 3). La cápsula endoscópica (CE) es una herramienta diagnóstica innovadora, tecnológica y no invasiva. Su principal utilidad es en el estudio de los trastornos del intestino delgado; aunque en años recientes también se han desarrollado tecnologías para el diagnóstico de entidades esofágicas y colónicas $(1,2)$. Fue introducida por primera vez al principio de los años 2000 (3). La capsula endoscópica es capaz de tomar imágenes con excelente resolución, recopilar videos de alta definición durante el paso por el sistema digestivo, almacenarlos y enviarlos al clínico a través de su interfaz digital (2).

\section{ASPECTOS TÉCNICOS}

La cápsula endoscópica es una herramienta de aproximadamente $30 \mathrm{~mm}$ de longitud y $12 \mathrm{~mm}$ de diámetro, que cuenta en su interior con varias cámaras con lente e iluminación propia $(4,5)$. Los lentes de las cámaras tienen una capacidad de magnificación de 1:8, inclusive mayor que la de los endoscopios normales. Cuenta con una memoria que permite almacenar las imágenes capturadas, y un software capaz de enviar los videos adquiridos a una computadora para su posterior análisis $(4,5)$.
Las aplicaciones de las cápsulas endoscópicas han ido creciendo desde su introducción. Dentro de las principales ventajas con respecto a la endoscopía son: presentar mayor adherencia por su naturaleza menos invasiva, ser un método no irradiante, ser de fácil utilización y acceso, no requerir anestesia ni sedación, y ser capaz de valorar la totalidad de la mucosa del intestino delgado. Cuenta con la ventaja de ser una "endoscopía fisiológica" ya que obtiene imágenes de una mucosa colapsada (no utiliza inflación intestinal) a través de los movimientos pasivos del intestino $(5,6)$. El éxito diagnóstico de la cápsula endoscópica se basa en la tasa de terminación del estudio, la calidad de la visibilidad del intestino delgado y el rendimiento diagnóstico (1). Actualmente se cuenta con 5 cápsulas para el estudio del intestino delgado (PillCam $\mathrm{SB} \AA$, Endocapsule $\AA$, MiRo capsule $\AA$, CapsoCam ® y OMOM®) y una cápsula para el estudio esofágico (PillCam ESO). En la TABLA 1 se enlistan algunas características de estas cápsulas $(6,7)$.

\section{INDICACIONES Y CONTRAINDICACIONES}

Las indicaciones formales de la cápsula endoscópica son: enfermedad inflamatoria intestinal (principalmente la enfermedad de Crohn por su compromiso del intestino delgado), enfermedad celiaca, sangrado digestivo de origen oculto, neoplasias del intestino 
TABLA 1. Diferencias de las cápsulas endoscópicas disponibles en el mercado $(2,6,7)$.

\begin{tabular}{|c|c|c|c|c|c|}
\hline & PillCam SB3® & EndoCapsule® & MiroCam ${ }^{\circledR}$ & CapsoCam Sv1® & OMOM® \\
\hline Empresa & Meditronic & Olympus & IntroMedic & CapsoVision & $\begin{array}{c}\text { Jinshan } \\
\text { Science and } \\
\text { Technology }\end{array}$ \\
\hline Tamaño (mm) & 26 & 26 & 24 & 31 & 25.4 \\
\hline Diámetro (mm) & 11 & 11 & 11 & 11 & 11 \\
\hline Peso (g) & 3.4 & 3.8 & 3.4 & - & 4. 5 \\
\hline $\begin{array}{l}\text { Toma de fotos } \\
\text { (fotos/s) }\end{array}$ & $2-6$ & 2 & 3 & $\begin{array}{l}\text { 12-20 (3-5 por } \\
\text { cámara) }\end{array}$ & $2-6$ \\
\hline Campo de visión & $156^{\circ}$ & $145^{\circ}$ & $150^{\circ}$ & $360^{\circ}$ & 165 \\
\hline Tiempo de video $(\mathrm{h})$ & 11 & 12 & 12 & 15 & 10 o más \\
\hline $\begin{array}{l}\text { Transmisión de la } \\
\text { imagen }\end{array}$ & Radiofrecuencia & Radiofrecuencia & $\begin{array}{c}\text { Comunicación } \\
\text { con cuerpo } \\
\text { humano }\end{array}$ & CapsoView & $\begin{array}{c}\text { Radiofrecuenc } \\
\text { ia }\end{array}$ \\
\hline
\end{tabular}

delgado, síndromes polipósicos hereditarios (síndrome de Peutz-Jeghers y adenomatosis polipósica familiar), tamizaje de malignización en pacientes portadores de síndrome de Lynch, monitorización de efectos adversos de fármacos, diarrea crónica y dolor abdominal $(2,4,6,8)$.

Debido a la naturaleza diagnóstica de la $\mathrm{CE}$, existen algunas contraindicaciones establecidas desde su amplio uso a nivel mundial. Algunas contraindicaciones son: el conocimiento o la sospecha clínica de obstrucción intestinal, los desórdenes del vaciamiento gástrico (p.e. gastroparesia), disfagia, embarazo, pacientes pediátricos menores de 2 años de edad, ser portador de un marcapasos cardiacos o cualquier dispositivo implantable, enfermedad epiléptica conocida, enfermedad cardiaca conocida, incapacidad para la comunicación (p.e. estados de demencia) y la incapacidad del centro médico para facilitar la extracción quirúrgica en caso de ser necesario. Además se debe evitar realizar resonancia magnética nuclear mientras el paciente se encuentra en estudio con la CE (1).

\section{PROCEDIMIENTO DIAGNÓSTICO Y PREPARACIÓN INTESTINAL}

El procedimiento diagnóstico se puede realizar de manera ambulatoria o intrahospitalaria; sin embargo para lograr un estudio óptimo se requiere de una adecuada preparación intestinal previa. El paciente debe iniciar una dieta con líquidos claros un día antes del procedimiento y debe tener un ayuno de al menos 12 horas previo a la ingesta de la CE $(3,9)$. Las guías coreanas recomiendan el uso de polietilenglicol (PEG) para aumentar la visibilidad del intestino delgado $y$ el rendimiento diagnóstico; sin embargo los últimos 
meta-análisis han demostrado que la utilidad del PEG es únicamente en la mejoría de la visibilidad del intestino delgado (2). A pesar que los proquinéticos aumentan el vaciamiento gástrico, no han demostrado mejorar el rendimiento diagnóstico ni tampoco un aumento en la tasa de terminación de estudios. Por lo anterior, actualmente no se recomienda su uso (2).

La cápsula endoscópica se ingiere por la vía oral con un vaso de agua. Una vez que el paciente ha ingerido la $C E$ es capaz de volver a realizar sus actividades diarias habituales. Con respecto a la alimentación, se permite la ingesta de líquidos claros dos horas posterior al ingreso de la CE y de una comida ligera 4 horas después de la ingesta de la CE. La cápsula endoscópica tendrá una salida natural a través de las heces (9).

Al ingresar al tracto digestivo, la cápsula endoscópica adquiere las imágenes a una velocidad de 2 a 6 imágenes por segundo, que posteriormente son transmitidas a la memoria $(6,8)$. Al final del estudio, se obtienen en promedio un total de 55000 imágenes en un periodo de 8 a 20 horas, que posteriormente son analizadas por el clínico a través del software. Tiene una tasa de detección global de patologías de alrededor del $60 \%$. Se han descrito pocas complicaciones y desventajas con el uso de la cápsula endoscópica. La principal complicación, aunque poco frecuente, es la retención de la CE que se define como la falla del dispositivo para llegar al colon mientras se encuentra encendida (2). También se han descrito otras complicaciones menos frecuentes como: la impactación en el musculo cricofaríngeo, la aspiración al tracto respiratorio, intervención quirúrgica para extracción de piezas por fractura de la $\mathrm{CE}$, perforación intestinal e impactación en un divertículo de Zenker. (2, 8, 9, 10, 11).

Por otro lado, la principal desventaja que ofrece el uso de la cápsula endoscópica es que es incapaz de realizar toma de biopsias o intervenciones terapéuticas (2, $8,9)$.

\section{PRINCIPALES ENTIDADES CLÍNICAS PARA SU USO COMO MÉTODO DIAGNÓSTICO}

En el 2015, Chavarría expuso un análisis de la utilización de las cápsulas endoscópicas en Costa Rica, en donde se expone que los principales motivos de referencia para la utilización de la misma fueron sangrado intestinal de origen desconocido, sospecha de enfermedad de Crohn y el estudio de anemia ferropénica (8). Los principales hallazgos endoscópicos en dicho estudio fueron angiodisplasias, ulceraciones de la mucosa y la ileitis ulcerativa (8). Por lo tanto, se expondrá la evidencia más reciente que existe entorno a la utilidad de la CE en esos tres escenarios clínicos.

\section{SANGRADO DIGESTIVO DE ORIGEN DESCONOCIDO}

El sangrado digestivo de origen desconocido se define como una entidad clínica en la que existe sangrado del tracto gastrointestinal persistente 0 recurrente sin que exista una etiología clara definida por esofagogastroduodenoscopía, colonoscopía o evaluación radiológica. En el curso clínico puede manifestarse 
con anemia ferropénica y/o sangre oculta en heces $(2,12)$.

En el manejo del sangrado digestivo de origen incierto, varias guías internacionales recomiendan la CE como primer estudio diagnóstico (13). Debe realizarse en los primeros 14 días desde el sangrado para maximizar el rendimiento diagnóstico (13). Actualmente es la principal indicación a nivel mundial para el uso de la CE como método diagnóstico en gastroenterología. Según un estudio publicado en México, las angiodisplasias fueron la principal causa de sangrado digestivo de origen desconocido obtenida por endoscopía capsular. En este mismo estudio, determinaron que el diagnóstico se obtuvo en un $80 \%$ de los casos con sangrado de origen evidente y en $62 \%$ de los casos de hemorragia de origen oculto (12).

Debido a que la CE no es capaz de tomar biopsias, su utilidad radica en la selección de la ruta más cercana para enteroscopía asistida por balón (2).

En un meta-análisis recientemente publicado, se evidenció que la precisión diagnóstica para detectar sangrado intestinal de origen vascular se aumentó al utilizar la cápsula endoscópica concomitantemente con la endoscopía asistida por balón (14).

\section{ENFERMEDAD DE CROHN (EC)}

Tanto las guías canadienses como europeas recomiendan la CE para pacientes con síntomas patognomónicos de enfermedad de Crohn que presenten ileocolonoscopía negativa y estudios radiológicos negativos $(2,13)$. Además su utilización se recomienda para pacientes con EC diagnosticada que tengan sintomatología incompatible con la endoscopía. También se encuentra indicada para determinar la cicatrización de la mucosa intestinal y la respuesta al tratamiento médico (15).

No se recomienda utilizar la $\mathrm{CE}$ en aquellos pacientes que tengan dolor abdominal crónico o diarrea sin la evidencia de elevación de biomarcadores. Tampoco se debe utilizar en aquellos que tengan estrecheces conocidas o ante la sospecha de un cuadro con estrecheces (2).

Las nuevas tecnologías orientan hacia la creación de una cápsula "small-bowel colon" (SBCE) para obtener imágenes de intestino delgado y colon. Recientemente se comparó el rendimiento diagnóstico de la ileocolonoscopía vs la SBCE para lesiones activas en enfermedad de Crohn. En este estudio se demostró que la SBCE tiene un rendimiento diagnóstico significativamente mayor que la ileocolonoscopía (2). En ese mismo estudio se demostró que de los doce pacientes que fueron catalogados positivos con la $\mathrm{CE}$, solo 3 fueron catalogados positivos por la ileocolonoscopía. Este estudio orienta a que el rendimiento diagnóstico de este tipo de cápsula pueda ser mayor que el de la ileocolonoscopía, sin embargo se requiere de más estudios para precisar su verdadero rendimiento diagnóstico (2).

\section{ANEMIA POR DEFICIENCIA DE HIERRO}

La anemia por deficiencia de hierro es la principal etiología de anemia a nivel mundial. La anemia ferropriva suele ser secundaria por sangrados intestinales.

La utilidad de la CE en contexto de anemia por deficiencia de hierro es más 
valioso en adultos mayores de 60 años. Este grupo etario presenta más hallazgos vasculares como principal etiología de la anemia por deficiencia de hierro. Además se ha descrito que el rendimiento diagnóstico en esta población se encuentra afectado por enfermedades prevalentes como enfermedad hepática crónica, enfermedades hematológicas, enfermedad renal crónica y el uso de anticoagulantes (16).

Por lo tanto, la CE tiene una utilidad diagnóstica en el escenario donde no se ha logrado determinar la etiología de la enfermedad por endoscopia digestiva o por colonoscopía. Se ha descrito un rendimiento diagnóstico del 57\% (2, 6, 16).

\section{NUEVAS APROXIMACIONES}

- CÁPSUlA ENDOSCÓPICA ESOFÁGICA (CEE)

Fue introducida por primera vez en el 2004. Tiene la capacidad de obtener 414 imágenes por segundo con sus dos cámaras en un lapso de 20 a 30 minutos. Se debe utilizar con el paciente en posición supina para aumentar la tasa de detección y otros parámetros estadísticos (17). Actualmente se encuentra disponible también la cápsula endoscópica para trastornos esofágicos. Su rol se encuentra limitado por su incapacidad para la toma de biopsias y para realizar procedimientos intervencionistas. Sin embargo permite la evaluación del paciente con pirosis, y para el tamizaje de varices esofágicas y de esófago de Barrett (con aprobación por FDA únicamente para los dos primeros escenarios clínicos). Su rendimiento diagnóstico la hace inferior a la endoscopía alta. Por lo tanto, la CE no reemplaza a la gastroscopía en la evaluación de trastornos esofágicos (17).

\section{- CÁPSUla ENDOSCÓPICA COLÓNICA (CEC)}

Fue introducida por primera vez en 2006. Requiere de preparación intestinal con dieta líquidos claros un día antes y la ingesta de $4 \mathrm{~L}$ de PEG durante la noche anterior y durante la mañana del estudio. Si bien la colonoscopía continúa siendo el gold standard para la detección de pólipos, la CEC tiene un papel importante en aquellos pacientes con colonoscopías incompletas $(13,18)$. Un estudio comparó la sensibilidad relativa de la CEC comparada con la colonografía por TAC (CTC). Se determinó un aumento significativo en la sensibilidad de lesiones de más de $6 \mathrm{~mm}$ y que además tiene mayores tasas de adherencia. La sensibilidad de la CEC para la detección de pólipos es de $88 \%$ cuando los pólipos superan los $6 \mathrm{~mm}$ de tamaño (18).

Actualmente se encuentra aprobada por la FDA y la EMA para el tamizaje de cáncer colónico en pacientes con endoscopías incompletas y para aquellos pacientes con alto riesgo anestésico. Sin embargo una vez más su utilidad se encuentra limitada por la incapacidad para toma de biopsia (18).

La CEC ha sido utilizada también para evaluar la actividad de la enfermedad de Crohn (13).

La CEC tiene una sensibilidad de $86 \%$ y $40 \%$ para detectar ulceraciones colónicas. Sin embargo, al compararlo con la colonoscopía usual, tiende a sobreestimar la actividad de la enfermedad (18). Por lo tanto, la Asociación Americana de 
Gastroenterólogos no recomienda la sustitución de la colonoscopía por la CEC para determinar la extensión y severidad de la enfermedad (18).

\section{CONCLUSIONES}

La cápsula endoscópica es una modalidad diagnóstica de gran utilidad en los desórdenes del intestino delgado. Es un estudio amigable con el paciente y ampliamente aceptada por el mismo debido a su facilidad, no invasión y fácil preparación intestinal.

Tiene alta eficacia diagnóstica en sangrado digestivo de origen indeterminado. Actualmente se encuentra en desarrollo la utilidad de la CE para otros trastornos del aparato digestivo.

\section{REFERENCIAS}

1. Bandorski D, Kurniawan N, Baltes P, Hoeltgen R, Hecker M, Stunder D, Keuchel M. Contraindications for video capsule endoscopy. World Journal of Gastroenterology. 2016; 22(45): 9898-9899. https://doi.org/10.3748/wjg.v22.i45.9898

2. Hosoe N, Takabayashi K, Ogata H, Kanai T. Capsule endoscopy for small-intestinal disorders: Current status. Digestive Endoscopy. 201902 10;. https://doi.org/10.1111/den.13346

3. Li Z, Liao Z, McAlindon M. Handbook of Capsule Endoscopy. Dordrecht: Springer; 2014. https://doi.org/10.1007/978-94-017-9229-5

4. Abstract cellular O'Loughlin CJ, Singh S, Lim R, Manten H. Localization of gastric bleeding with wireless capsule endoscopy - the advantages of physiologic endoscopy. American Journal of Gastroenterology. 2003 Set; 98(9): S144. Disponible en: http://www.readcube.com/articles/10.1111/j.1572$\underline{0241.2003 .08159 . x}$

5. Marshall CA, Cave DR. Preparation for video capsule endoscopy: A clear choice?. Gastrointestinal Endoscopy. 2017 01;85(1):194-195. https://doi.org/10.1016/j.gie.2016.08.037

6. Redondo-Cerezo E. Wireless capsule endoscopy: Perspectives beyond gastrointestinal bleeding. World Journal of Gastroenterology. 2014;20(42):15664-16673. https://doi.org/10.3748/wjg.v20.i42.15664

7. Scott R, Enns R. Advances in Capsule Endoscopy. Gastroenterology \& Hepatology. 2015 Set 9; 11 (9): 612-619. Disponible en: https://www.ncbi.nlm.nih.gov/pmc/articles/PMC4965621/

8. Chavarría I. Análisis de la utilización de las capsulas endoscópicas en el Hospital San Juan de Dios de febrero de 2012 a marzo de 2015: Características de los pacientes, tasas de finalización y rendimiento [Tesis]. Universidad de Costa Rica; $2015 . \quad$ Disponible en: http://repositorio.sibdi.ucr.ac.cr:8080/jspui/handle/123456789/3156

9. Wang A, Banerjee S, Barth BA, Bhat YM, Chauhan S, Gottlieb KT, Konda V, Maple JT, Murad F, Pfau PR, Pleskow DK, Siddiqui UD, Tokar JL, Rodriguez SA. Wireless capsule endoscopy. Gastrointestinal Endoscopy. 2013 Dec;78(6):805-815. https://doi.org/10.1016/j.gie.2013.06.026

10. Sánchez-Chávez X, Martínez-García C. Aspiración de cápsula endoscópica: ¿complicación frecuente? $\begin{array}{lllll}\text { Revista de Gastroenterología de } 2013 & \text { Oct;78(4):257-258. }\end{array}$ https://doi.org/10.1016/i.rgmx.2013.04.003 
11. González-Suárez B, Galter S, Balanzó J. Cápsula endoscópica: fundamentos y utilidad clínica. Cirugía Española. 2007 06; 81(6): 299-306. https://doi.org/10.1016/s0009-739x(07)71328-3

12. Hernández-López A, Arano-Acua J, Gálvez-Ríos S, Meixueiro-Daza A, Reyes-Huerta J, de la Cruz-Patiño E, Roesch-Dietlen F, Remes-Troche J. Experiencia clínica con el uso de 2 sistemas de cápsula endoscópica en el diagnóstico de enfermedades del intestino delgado. Endoscopia. 2014 01;26(1):12-18. https://doi.org/10.1016/i.endomx.2014.04.003

13. Spada C, Hassan C, Galmiche J, Neuhaus H, Dumonceau J, Adler S, Epstein O, Gay G, Pennazio M, Rex D, Benamouzig R, de Franchis R, Delvaux M, Devière J, Eliakim R, Fraser C, Hagenmuller F, Herrerias J, Keuchel M, Macrae F, Munoz-Navas M, Ponchon T, Quintero E, Riccioni M, Rondonotti E, Marmo R, Sung J, Tajiri H, Toth E, Triantafyllou K, Van Gossum A, Costamagna G. Colon capsule endoscopy: European Society of Gastrointestinal Endoscopy (ESGE) Guideline. Endoscopy. 201203 02;44(05):527536. https://doi.org/10.1055/s-0031-1291717

14. Brito HP, Ribeiro IB, Moura DTHD, Bernardo WM, Chaves DM, Kuga R, Maahs ED, Ishida RK, Moura ETHD, Moura EGHD. Video capsule endoscopy vs double-balloon enteroscopy in the diagnosis of small bowel bleeding: A systematic review and meta-analysis. World Journal of Gastrointestinal Endoscopy. 2018 Dec 16;10(12):400-421. https://doi.org/10.4253/wige.v10.i12.400

15. Goran L, Negreanu AM, Stemate A, Negreanu L. Capsule endoscopy: Current status and role in Crohn's disease. World Journal of Gastrointestinal Endoscopy. 201809 16;10(9):184-192. https://doi.org/10.4253/wjge.v10.i9.184

16. Xavier S, Magalhães J, Rosa B, Moreira MJ, Cotter J. Impact of small bowel capsule endoscopy in iron deficiency anemia: influence of patient'sage on diagnostic yield. Arquivos de Gastroenterologia. 2018 09;55(3):242-246. https://doi.org/10.1590/s0004-2803.201800000-61

17. Park J, Cho YK, Kim JH. Current and Future Use of Esophageal Capsule Endoscopy. Clinical Endoscopy. 201807 31;51(4):317-322. https://doi.org/10.5946/ce.2018.101

18. Hong SN, Kang S, Jang HJ, Wallace MB. Recent Advance in Colon Capsule Endoscopy: What's New?. Clinical Endoscopy. $2018 \quad 07 \quad 31 ; 51(4): 334-343 . \quad \underline{\text { https://doi.org/10.5946/ce.2018.121 }}$ 